\title{
Genetic basis of benzimidazole resistance in Teladorsagia circumcincta in Ireland
}

\author{
Jason D. Keegan ${ }^{1,3}$, Barbara Good ${ }^{2}$, Theo de Waal ${ }^{3}$, June Fanning ${ }^{4}$ and Orla M. Keane ${ }^{1^{*}}$
}

\begin{abstract}
Resistance to benzimidazole (BZ) anthelmintics is common in ovine nematodes of economic importance. Single nucleotide polymorphisms (SNP) at three positions in the isotype $1 \beta$ - tubulin gene have been associated with BZ resistance and molecular tests for the detection of BZ resistance have been developed. In order to determine if such tests are practicable in Ireland the polymorphisms associated with BZ resistance must be identified. To this end, BZresistant nematodes were recovered from four farms in Ireland. Resistant Teladorsagia circumcincta, Cooperia curticei and Trichostrongylus colubriformis were recovered, with resistant T. circumcincta the most common and the only species studied further. Sequencing of the isotype $1 \beta$-tubulin gene from resistant $T$. circumcincta identified a T - A transition, resulting in an F200Y substitution known to be responsible for BZ-resistance, on three of the farms. However, on the fourth farm the frequency of the resistant A allele was only 0.33 indicating another BZ resistance mechanism may be present on this farm. An additional polymorphism resulting in a substitution of glutamate for leucine (E198L) was also found on this farm at low frequency (0.17). No polymorphisms at position 167 were identified on any farm. Therefore, molecular tests to detect BZ resistance in T. circumcincta in Ireland could prove useful; however, they may result in some instances of resistance remaining undetected.
\end{abstract}

Keywords: Sheep, Anthelmintic, Resistance, Benzimidazole, Teladorsagia, $\beta$-tubulin

\section{Introduction}

Anthelmintic drugs are heavily relied upon to control the effects of parasitic helminths in sheep, but their overuse has led to the development of anthelmintic resistance (AR). Resistance to benzimidazole (BZ) on Irish sheep farms has been reported [1-3] but the prevalence may be underestimated, given the insensitivity of diagnosing AR based on faecal egg count [4]. In BZsusceptible nematodes, $\mathrm{BZ}$ binds to tubulin disrupting the formation of microtubules, resulting in a reduction in glucose uptake and protein secretion, which leads to starvation and death. In resistant nematodes, an amino acid change in the tubulin protein prevents BZ from binding [5]. Three non-synonymous single nucleotide polymorphisms (SNP) in the isotype $1 \beta$-tubulin gene have been associated with $\mathrm{BZ}$ resistance in a variety of ovine gastrointestinal nematode (GIN) species. The most common of these polymorphisms results in a phenylalanine to tyrosine substitution at position 200 (F200Y), while the other SNP result in a phenylalanine to tyrosine substitution at position 167 (F167Y) or a glutamic acid to alanine substitution at position 198 (E198A) [6-8]. However, BZ resistant nematodes have been found without any of the three known mutations, suggesting the existence of additional determinants of resistance [9]. The polymorphisms responsible for $\mathrm{BZ}$ resistance in Irish ovine nematodes remain unknown.

Recently molecular tests for the detection of BZ resistance in a variety of ovine nematode species have been developed $[7,10,11]$. The objective of this study was to investigate the molecular basis for BZ resistance in Irish field populations of Teladorsagia circumcincta, one of the most common GIN species found in sheep in Ireland $[12,13]$, with a view to establishing whether such molecular methods of BZ-resistance diagnosis are practicable in Ireland.

\footnotetext{
* Correspondence: orla.keane@teagasc.ie

${ }^{1}$ Animal and Bioscience Department, Teagasc, Grange, Dunsany, Co. Meath, Ireland

Full list of author information is available at the end of the article
} 


\section{Materials and methods Nematode isolation}

Four geographically dispersed farms (Wicklow, Kerry, Roscommon and Galway) with a confirmed history of BZ resistance were recruited [1]. Four lambs were purchased from each farm and brought to the Department of Agriculture, Food and the Marine (DAFM) farm in Co. Kildare. The lambs were housed in quarantine with straw bedding as required. Lambs were fed hay and a commercial pelleted ration with free access to water. Lambs were given 2 days to acclimatise before faecal samples were collected per rectum and faecal egg count (FEC) determined using the modified McMaster method with a minimum sensitivity of 25 eggs per gram (epg) [14]. Lambs were subsequently treated with a BZ product (Systamex ${ }^{\oplus}, 5 \mathrm{mg} / \mathrm{kg}$ per os (p.o.), Schering Plough) as per manufacturer's instructions. Fourteen days posttreatment a faecal samples was collected and FEC determined and lambs were treated a second time with the same anthelmintic product at the same dose rate. Ten days (farms $\mathrm{A}$ and B) or 13 days (farms $\mathrm{C}$ and $\mathrm{D}$ ) after the second BZ treatment, faecal samples were collected and FEC determined as described above in order to calculate the reduction in egg count following the second treatment. The lambs were euthanised 14-16 days after the second BZ treatment and the gastrointestinal tract removed. The abomasum was processed to recover GIN according to the method previously described [15] with the exception that GIN were stored in water after washing; while the first $10 \mathrm{~m}$ of the small intestine was processed to recover GIN as described previously [16]. Adult male nematodes were speciated based on their morphological features [14] and live worms were preserved individually in RNAlater or PBS and frozen at $-20{ }^{\circ} \mathrm{C}$. For each farm, faecal egg count reductions were calculated as ((mean FEC pre-treatment - mean FEC post 2nd treatment)/mean FEC pre-treatment)*100.

DNA extraction and isotype $1 \boldsymbol{\beta}$-tubulin gene sequencing DNA was extracted from individual adult male $T$. circumcincta using the Qiagen QIAmp ${ }^{\oplus}$ DNA micro kit as described in the manufacturer's protocol. DNA was eluted in $15 \mu \mathrm{L}$ of nuclease free water and preserved at $-20{ }^{\circ} \mathrm{C}$. Initially the full 4216 bp isotype $1 \beta$-tubulin gene was amplified in a reaction containing $0.5 \mu \mathrm{M}$ Tcirc_btubulinF and Tcirc_btubulinR primers (Table 1), $200 \mu \mathrm{M}$ dNTPs, $1 \times$ High Fidelity buffer, 1 unit of Phusion high fidelity DNA polymerase (New England Biolabs, UK) and $1.5 \mu \mathrm{L}$ of template DNA in a $50 \mu \mathrm{L}$ reaction. The reaction was denatured at $98{ }^{\circ} \mathrm{C}$ for $2 \mathrm{~min}$ followed by 30 cycles of $98{ }^{\circ} \mathrm{C}$ for $10 \mathrm{~s} ; 64.9{ }^{\circ} \mathrm{C}$ for $10 \mathrm{~s}$ and $72{ }^{\circ} \mathrm{C}$ for $2 \mathrm{~min}$ with a final extension step for $72{ }^{\circ} \mathrm{C}$ for $5 \mathrm{~min}$. Subsequently another PCR was designed to amplify the $\beta$-tubulin gene in two $\sim 2 \mathrm{~kb}$ fragments
Table 1 Primer sequences for Teladorsagia circumcincta isotype $1 \beta$-tubulin amplification and sequencing

\begin{tabular}{lll}
\hline Primer Name & Function & Sequence 5' 3 $^{\prime}$ \\
\hline Tcirc_btubulinF & amplification & CCTCGACTACAATCATGCGTG \\
Tcirc_btubulinR & amplification & CGCAACCAATGTGTATTCG \\
Tcirc_btubulin_intF & amplification & GGACAACTITCCGTCCAGA \\
TCirc_btubulin_intR & amplification & CAACCCTCTGCCTCTTTACG \\
TCSQ1R & sequencing & AGCCAGGACAGAGAACAACG \\
TCSQ2F & sequencing & AGTCCAGGAGAGGGGAAAA \\
TCSQ3F & sequencing & AATGCGAAAGTGCTTCACCT \\
TCSQ4R & sequencing & AACTGTCCAGGGAATCGAAG \\
TCSQ5F & sequencing & TGCTTCCGCACCTTAAAACT \\
TCSQ6F & sequencing & CCCACGACTGCTTTGTGTAA \\
\hline
\end{tabular}

(Tcirc_btubulinF and Tcirc_btubulin_intR; Tcirc_btubuli$\mathrm{n}$ intF and Tcirc_btubulinR; Table 1), using the same conditions as above except with an elongation time of $1 \mathrm{~min}$. Cloning of the $4216 \mathrm{bp}$ PCR product was also conducted for 8 nematodes as direct sequencing of the PCR products was found to be unreliable. The PCR product was gel purified using the QIAquick PCR purification kit (Qiagen, Germany) and cloned using the TOPO XL cloning kit as per manufacturer's instructions. Between 1 and 4 plasmid inserts per individual nematode were sequenced. The amplification and sequencing primers used are listed in Table 1. Purified PCR products or plasmids were sent for Sanger sequencing at Source Bioscience (Waterford, Ireland) or Beckman Coulter Genomics (Essex, UK). Six sequencing primers were used to obtain the entire sequence including the introns. Sequences were assembled in bioedit using cap3 sequence assembly software [17] to generate the complete $\beta$-tubulin sequence per individual. Sequences were aligned in MEGA 5 using MUSCLE [18] and compared to the $T$. circumcincta mRNA for $\beta$-tubulin sequence (GenBank ID: Z69258.1) in order to identify coding sequence SNP.

\section{Results}

Faecal egg count reductions calculated from four lambs from each farm indicated BZ resistance on each farm with nematodes surviving two rounds of BZ treatment. BZ resistant $T$. circumcincta was recovered post-mortem from lambs from all four farms and was the only species present post-treatment on all farms tested. C. curticei was recovered post-mortem from lambs from three farms while $T$. colubriformis was recovered from lambs from two farms (Table 2). Only $T$. circumcincta was studied further.

The full sequence for the isotype $1 \beta$-tubulin gene was obtained for 36 individual male $T$. circumcincta (Wicklow $n=9$; Galway $n=8$; Roscommon $n=7$; and Kerry $n=12$ ). 
Table 2 Faecal egg count, species surviving benzimidazole treatment (s) and substitution frequencies at positions 198 and 200 of the $\beta$-tubulin protein for each of the farms

\begin{tabular}{|c|c|c|c|c|c|c|c|c|c|}
\hline \multirow[b]{2}{*}{ Farm } & \multicolumn{4}{|c|}{ Faecal egg count ${ }^{a}$} & \multicolumn{3}{|c|}{ Species recovered post-mortem } & \multicolumn{2}{|c|}{$\begin{array}{l}\text { Substitution } \\
\text { frequency }\end{array}$} \\
\hline & $\begin{array}{l}\text { Pre-treatment } \\
\text { (epg) }\end{array}$ & $\begin{array}{l}\text { Post } 1^{\text {st }} \\
\text { treatment (epg) }\end{array}$ & $\begin{array}{l}\text { Post 2 }{ }^{\text {nd }} \\
\text { treatment (epg) }\end{array}$ & $\begin{array}{l}\% \\
\text { Reduction }\end{array}$ & $\begin{array}{l}\text { Teladorsagia } \\
\text { circumcincta }\end{array}$ & $\begin{array}{l}\text { Cooperia } \\
\text { curticei }\end{array}$ & $\begin{array}{l}\text { Trichostrongylus } \\
\text { colubriformis }\end{array}$ & E198L & F200Y \\
\hline Wicklow & 1370 & 568 & 794 & 42 & + & + & - & 0.17 & 0.33 \\
\hline Kerry & 889 & 409 & 406 & 54 & + & + & - & - & 1 \\
\hline Roscommon & 1050 & 647 & 469 & 55 & + & + & + & - & 1 \\
\hline Galway & 1122 & 263 & 194 & 83 & + & - & + & - & 0.94 \\
\hline
\end{tabular}

${ }^{\text {a }}$ represents the average of 4 lambs per farm

breduction calculated after 2 benzimidazole treatments

The most common polymorphism was a $\mathrm{T}$ to $\mathrm{A}$ transition (TTC - TAC) resulting in a phenylalanine to tyrosine substitution at codon 200 (F200Y). This polymorphism had an overall frequency of 0.79 among the worms which survived two rounds of BZ treatment. However, the frequency differed between the farms, with the polymorphism fixed in the worms from Kerry and Roscommon, with all individuals TAC/TAC homozygotes. On the Galway farm, 7 of the surviving nematodes were homozygous for the polymorphism with the remaining individual heterozygous (frequency $=0.94$ ). However, on the Wicklow farm the frequency of TAC was only 0.33 , with only one $T$. circumcincta which survived BZ treatment homozygous for the polymorphism. A further four surviving individuals were heterozygous while the remaining four individuals had the BZ susceptible genotype (TTC). Interestingly, two nematodes from the farm in Wicklow encoded nonsynonymous substitutions at position 198 resulting in a substitution of glutamate for leucine (E198L). One of these nematodes was homozygous for the TTC BZ susceptible allele at position 200, with the other heterozygous. No other polymorphisms at codon 198 were detected and there were no polymorphisms at codon 167 either.

\section{Discussion}

Knowledge of the mechanisms contributing to AR can help inform strategies designed to control the development and spread of AR. While BZ resistance among ovine nematodes in Ireland is considered common [1$3]$, information regarding the polymorphisms associated with BZ resistance in Ireland has been lacking. Previous studies indicated Teladorsagia spp., Cooperia spp. and Trichostrongylus spp., as the predominant BZ resistance genera [1]. The results of this study are in agreement with these findings. T. circumcincta was identified as the most common BZ resistant species, being found on all four farms.

Molecular detection of anthelmintic resistance is desirable as it can provide a more sensitive method of detection than conventional methods such as the faecal egg count reduction test [11]. However, as molecular anthelmintic resistance detection methods are developed it is important they are validated for use in different populations. The most commonly reported indicator of $\mathrm{BZ}$ resistance in ovine nematodes is a $\mathrm{T}$ to $\mathrm{A}$ transition in the isotype $1 \beta$-tubulin gene resulting in an F200Y substitution in the encoded protein. In agreement with previous studies, the F200Y substitution was evident in the majority (32/36) of BZ resistant $T$. circumcincta individuals in this study. It has been reported that this mutation is recessive in $T$. circumcincta and that only homozygous $\mathrm{Y} / \mathrm{Y}$ individuals survive $\mathrm{BZ}$ treatment [19]. In agreement with this, all BZ surviving nematodes from two farms were homozygous for the mutation. However, on the Galway farm, in addition to the 7 nematodes homozygous for the mutation, one F/Y heterozygote was recovered. Interestingly, only one nematode from Wicklow was homozygous for the mutation with four heterozygous and four homozygous for the wild-type allele. A nonsynonymous mutation at codon 198 (E198L) was also found in two worms from Wicklow. This E198L mutation has been reported to be associated with $\mathrm{BZ}$ resistance in $T$. circumcincta populations in the United Kingdom [20]. The amino acid at this position may be important in the mechanism of action of BZ as the E198A mutation has been experimentally shown to confer a greater level of resistance than the F200Y mutation in $H$. contortus [21]. However, the role of the E198L mutation in anthelmintic resistance remains to be definitely determined. In the Wicklow worm population, the frequency of the TAC polymorphism, found at amino acid position 200 and associated with resistance, was 0.33; indicating another $\mathrm{BZ}$ resistance mechanism may be present on this farm. Studies with $H$. contortus have indicated that the isotype- $2 \beta$-tubulin genes may also be of interest in terms of additional $\mathrm{BZ}$ resistance mechanisms [22]. Furthermore, other mechanisms of BZ resistance are suspected with the P-glycoproteins also implicated [23].

In agreement with other studies, the F200Y substitution resulting from a mutation in the isotype $1 \beta$-tubulin coding gene was the most commonly observed mutation in BZ-resistant $T$. circumcincta in Ireland. In light of these findings, application of molecular tests to detect 
BZ resistance in $T$. circumcincta could prove useful, however, caution is warranted as molecular tests based on known substitutions have the potential to leave some instances of resistance undetected.

\section{Abbreviations}

AR: Anthelmintic resistance; BZ: Benzimidazole; DAFM: Department of Agriculture Food and the Marine; EPG: Eggs per gram; FEC: Faecal egg count; GIN: Gastrointestinal nematode; PCR: Polymerase chain reaction; SNP: Single nucleotide polymorphism

\section{Acknowledgements}

The authors wish to acknowledge the cooperation of the farmers and the assistance of DAFM and Teagasc farm and technical staff.

\section{Funding}

The survey was funded by the DAFM Research Stimulus Fund via the EMIDAERA net project Coping with Anthelmintic Resistance in Ruminants (CARES) grant reference 11/RD/EMIDA/2.

\section{Availability of data and materials}

The datasets and material generated during this study are available from the corresponding author where possible.

\section{Authors' contributions}

BG JF and OMK coordinated the project. All authors contributed to study design. JDK performed sample collection and all laboratory work. All authors participated in manuscript preparation and read and approved the final manuscript.

\section{Authors' information}

JDK: B.A.(mod), M.Sc, Ph.D. BG: B.A.(mod), P.Grad.Dip., Ph.D., Senior Researcher in Parasitology, Teagasc. TDW: B.V.Sc., Ph.D., DipDatMet, HDipUTL, DipEVPC, MRCVS, Senior Lecturer and European Veterinary Specialist in Parasitology, UCD. JF: B.V.Sc., Ph.D., Research Officer, DAFM.OMK: B.A.(mod), P.Grad.Dip., Ph.D., Senior Researcher Teagasc

\section{Competing interests}

The authors declare that they have no competing interests.

\section{Consent for publication}

Not applicable.

\section{Ethics approval and consent to participate}

The euthanisation of lambs harbouring BZ resistant nematodes was conducted under experimental license (B100/3307) from the Department of Health, Ireland in accordance with the Cruelty to Animals Act, 1876 and the European Communities (Amendments of the Cruelty to Animals Act, 1876) Regulations, 2002, 2005

\section{Author details}

${ }^{1}$ Animal and Bioscience Department, Teagasc, Grange, Dunsany, Co. Meath, Ireland. ${ }^{2}$ Animal \& Bioscience Dept, Teagasc, Mellows Campus, Athenry, Co. Galway, Ireland. ${ }^{3}$ School of Veterinary Medicine, University College Dublin, Belfield, Dublin 4, Ireland. ${ }^{4}$ Department of Agriculture Food and the Marine, Central Veterinary Research Laboratory, Backweston, Co. Kildare, Ireland.

Received: 23 December 2016 Accepted: 9 February 2017

Published online: 13 February 2017

\section{References}

1. Good B, Hanrahan JP, de Waal DT, Patten T, Kinsella A, et al. Anthelminticresistant nematodes in Irish commercial sheep flocks- the state of play. Ir Vet J. 2012;65:21

2. Keane OM, Keegan JD, Good B, De Waal T, Fanning J, et al. High level of treatment failure with commonly used anthelmintics on Irish sheep farms. Ir Vet J. 2014;67:16

3. McMahon C, Bartley DJ, Edgar HW, Ellison SE, Barley JP, et al. Anthelmintic resistance in Northern Ireland (I): prevalence of resistance in ovine gastrointestinal nematodes, as determined through faecal egg count reduction testing. Vet Parasitol. 2013;195:122-30.

4. Martin PJ, Anderson N, Jarrett RG. Detecting benzimidazole resistance with faecal egg count reduction tests and in vitro assays. Aust Vet J. 1989:66:236-40.

5. Wolstenholme AJ, Fairweather I, Prichard R, von Samson-Himmelstjerna G, Sangster NC. Drug resistance in veterinary helminths. Trends Parasitol. 2004;20:469-76.

6. Chaudhry U, Redman EM, Raman M, Gilleard JS. Genetic evidence for the spread of a benzimidazole resistance mutation across southern India from a single origin in the parasitic nematode Haemonchus contortus. Int J Parasitol. 2015;45:721-8.

7. Morrison AA, Mitchell S, Mearns R, Richards I, Matthews JB, et al. Phenotypic and genotypic analysis of benzimidazole resistance in the ovine parasite Nematodirus battus. Vet Res. 2014:45:116.

8. Skuce P, Stenhouse L, Jackson F, Hypsa V, Gilleard J. Benzimidazole resistance allele haplotype diversity in United Kingdom isolates of Teladorsagia circumcincta supports a hypothesis of multiple origins of resistance by recurrent mutation. Int J Parasitol. 2010;40:1247-55.

9. Stenhouse LJ. Characterisation of anthelmintic resistance in a multiple drug resistant Teladorsagia circumcincta isolate. Glasgow: PhD thesis University of Glasgow; 2007.

10. Martinez-Valladares M, Donnan A, Geldhof P, Jackson F, Rojo-Vazquez FA, et al. Pyrosequencing analysis of the beta-tubulin gene in Spanish Teladorsagia circumcincta field isolates. Vet Parasitol. 2012;184:371-6.

11. von Samson-Himmelstjerna G, Walsh TK, Donnan AA, Carriere S, Jackson $F$, et al. Molecular detection of benzimidazole resistance in Haemonchus contortus using real-time PCR and pyrosequencing. Parasitology. 2009;136:349-58.

12. Good B, Grennan E, Crowley BA, Hanrahan JP. Effect of Grazing system and anthelmintic treatment of ewes on parasite challenge and lamb growth. Teagasc. 2001;4471:1-34

13. Good B, Hanrahan JP, Crowley BA, Mulcahy G. Texel sheep are more resistant to natural nematode challenge than Suffolk sheep based on faecal egg count and nematode burden. Vet Parasitol. 2006;136:317-27.

14. MAFF. Manual of Veterinary Parasitology Laboratory Techniques. Reference Book 418: Ministry of Agriculture, Fisheries and Food. 3rd ed. London: HMSO; 1986.

15. McRae KM, Good B, Hanrahan JP, Glynn A, O'Connell MJ, et al. Response to Teladorsagia circumcincta infection in Scottish Blackface lambs with divergent phenotypes for nematode resistance. Vet Parasitol. 2014;206:200-7.

16. Wood IB, Amaral NK, Bairden K, Duncan JL, Kassai T, et al. World Association for the Advancement of Veterinary Parasitology (W.A.A.V.P.) second edition of guidelines for evaluating the efficacy of anthelmintics in ruminants (bovine, ovine, caprine). Vet Parasitol. 1995;58:181-213.

17. Huang X, Madan A. CAP3: A DNA sequence assembly program. Genome Res. 1999;9:868-77.

18. Edgar RC. MUSCLE: multiple sequence alignment with high accuracy and high throughput. Nucleic Acids Res. 2004;32:1792-7.

19. Elard L, Humbert JF. Importance of the mutation of amino acid 200 of the isotype 1 beta-tubulin gene in the benzimidazole resistance of the smallruminant parasite Teladorsagia circumcincta. Parasitol Res. 1999;85:452-6.

20. Redman E, Whitelaw F, Tait A, Burgess C, Bartley Y, et al. The emergence of resistance to the benzimidazole anthlemintics in parasitic nematodes of livestock is characterised by multiple independent hard and soft selective sweeps. PLoS Negl Trop Dis. 2015;9:e0003494.

21. Kotze AC, Cowling K, Bagnall NH, Hines BM, Ruffell AP, et al. Relative level of thiabendazole resistance associated with the E198A and F200Y SNPs in larvae of a multi-drug resistant isolate of Haemonchus contortus. Int J Parasitol Drugs Drug Resist. 2012;2:92-7.

22. Saunders GI, Wasmuth JD, Beech R, Laing R, Hunt M, et al. Characterization and comparative analysis of the complete Haemonchus contortus betatubulin gene family and implications for benzimidazole resistance in strongylid nematodes. Int J Parasitol. 2013;43:465-75.

23. Blackhall WJ, Prichard RK, Beech RN. P-glycoprotein selection in strains of Haemonchus contortus resistant to benzimidazoles. Vet Parasitol. 2008;152:101-7. 